\title{
Obstructive Left Colon Cancer Should Be Managed by Using a Subtotal Colectomy Instead of Colonic Stenting
}

\author{
Chung Ki Min, Hyung Ook Kim, Donghyoun Lee, Kyung Uk Jung, Sung Ryol Lee, Hungdai Kim, \\ Ho-Kyung Chun \\ Department of Surgery, Kangbuk Samsung Hospital, Sungkyunkwan University School of Medicine, Seoul, Korea
}

Purpose: This study compared a subtotal colectomy to self-expandable metallic stent (SEMS) insertion as a bridge to surgery for patients with left colon-cancer obstruction.

Methods: Ninety-four consecutive patients with left colon-cancer obstruction underwent an emergency subtotal colectomy or elective SEMS insertion between January 2007 and August 2014. Using prospectively collected data, we performed a retrospective comparative analysis on an intention-to-treat basis.

Results: A subtotal colectomy and SEMS insertion were attempted in 24 and 70 patients, respectively. SEMS insertion technically failed in 5 patients $(7.1 \%)$. The mean age and rate of obstruction in the descending colon were higher in the subtotal colectomy group than the SEMS group. Sex, underlying disease, American Society of Anesthesiologists physical status, and pathological stage showed no statistical difference. Laparoscopic surgery was performed more frequently in patients in the SEMS group (62 of 70, 88.6\%) than in patients in the subtotal colectomy group (4 of 24, 16.7\%). The overall rate of postoperative morbidity was higher in the SEMS group. No Clavien-Dindo grade III or IV complications occurred in the subtotal colectomy group, but 2 patients (2.9\%) died from septic complications in the SEMS group. One patient $(4.2 \%)$ in the subtotal colectomy group had synchronous cancer. The total hospital stay was shorter in the subtotal colectomy group. The median number of bowel movements in the subtotal colectomy group was twice per day at postoperative 3-6 months.

Conclusion: A subtotal colectomy for patients with obstructive left-colon cancer is a clinically and oncologically safer, 1 -stage, surgical strategy compared to SEMS insertion as a bridge to surgery.

Keywords: Intestinal obstruction; Colon neoplasms; Colectomy; Self-expandable metallic stents

\section{INTRODUCTION}

Despite the availability of colorectal cancer screening programs,

Received: June 27, 2016 - Accepted: October 24, 2016

Correspondence to: Hyung Ook Kim, M.D.

Department of Surgery, Kangbuk Samsung Hospital, Sungkyunkwan

University School of Medicine, 29 Saemunan-ro, Jongno-gu, Seoul 03181, Korea

Tel: +82-2-2001-8540, Fax: +82-2-2001-2131,

E-mail: ho115.kim@samsung.com

This paper was presented as a poster at the meeting of the 48th Korean Society of Coloproctology Annual Meeting, Busan, Korea, April 10-12, 2015.

(C) 2016 The Korean Society of Coloproctology

This is an open-access article distributed under the terms of the Creative Commons Attribution NonCommercial License (http://creativecommons.org/licenses/by-nc/4.0) which permits unrestricted noncommercial use, distribution, and reproduction in any medium, provided the original work is properly cited.
$8 \%-29 \%$ of patients with colorectal cancer present with acute colonic obstruction [1]. Right colon-cancer obstruction is less common than left obstruction and is typically treated by using a right hemicolectomy or an extended right hemicolectomy [2]. Left colon and rectosigmoid junction cancers tend to obstruct earlier due to the smaller size of the lumen, and various surgical procedures have been used to treat them.

A single-stage resection and primary anastomosis can be performed with or without intraoperative colonic lavage, but surgical complications, including anastomotic leak, are common [3-5]. Preoperative placement of a self-expandable metallic stent (SEMS) as a bridge to surgery is used to avoid a Hartmann procedure or colostomy $[4,6,7]$. Of the various single-stage surgical techniques, the Hartmann procedure or colostomy has been suitable for patients with precarious general conditions. However, colonic stents have several disadvantages. The technical and clinical fail- 
ure rate of colonic SEMS is $20 \%-30 \%$ [4, 7-9] and a randomized controlled trial reported a technical failure rate of 53.3\% [10]. Some reports suggest that SEMS insertion is associated with adverse oncological outcomes [11-13]. Furthermore, most surgeons usually perform elective surgery 5-10 days after SEMS insertion $[6,7,14]$; thus, the interval between SEMS insertion and surgery may increase the total hospital stay.

A subtotal colectomy is suggested as an effective surgical option for treating patients with left colon-cancer obstruction $[3,5]$. Ileosigmoid anastomosis after a subtotal colectomy for patients with colon cancer results in good bowel function and quality of life [15]. A subtotal colectomy can remove synchronous proximal neoplasms and reduce the risk of subsequent metachronous tumor development $[14,16]$. However, until now, no study has exclusively compared a subtotal colectomy to SEMS insertion as a bridge to surgery for patients with left colon-cancer obstruction, although one study included subtotal and total colectomies [14]. A total colectomy with ileorectal anastomosis leads to a deterioration in functional outcomes, including frequent bowel movement. Therefore, we compared only the subtotal colectomy to SEMS insertion as a bridge to surgery for patients with left colon-cancer obstruction based on morbidity, mortality, bowel function, and hospital stay.

\section{METHODS}

This study enrolled 94 consecutive patients with left colon-cancer obstruction who received an emergency subtotal colectomy or SEMS insertion as elective surgery between January 2007 and August 2014 at Kangbuk Samsung Hospital, Sungkyunkwan University School of Medicine, Seoul, Korea. Inclusion criteria were reasonable operative risk, resectable acutely obstructive descending or sigmoid colon cancer, and massively distended proximal colon suggesting complete or near complete obstruction (Fig. 1). Patients with rectal or rectosigmoid junction cancer were excluded because they would need a total colectomy or 2-stage surgery without a method to bridge to elective surgery, such as SEMS or intraoperative colonic irrigation. Patients who received a colon segmental resection without SEMS were also excluded. Other exclusion criteria were obstruction proximal to splenic flexure or pneumoperitoneum or septic condition on emergency room arrival, which generally required a staged surgery using colostomy. Using prospectively collected data, we performed a retrospective comparative analysis after approval (KBSMC 2016-08-017) by the ethics committee at Kangbuk Samsung Hospital.

In our department, surgery type is generally based on surgeon preference and intraoperative findings. One surgeon performed a subtotal colectomy in most cases with left colon-cancer obstruction, regardless of the presence or absence of proximal colonic ischemia; other surgeons usually perform SEMS insertion as a bridge to surgery or a colon segmental resection with intraoperative colonic manual decompression. A subtotal colectomy was
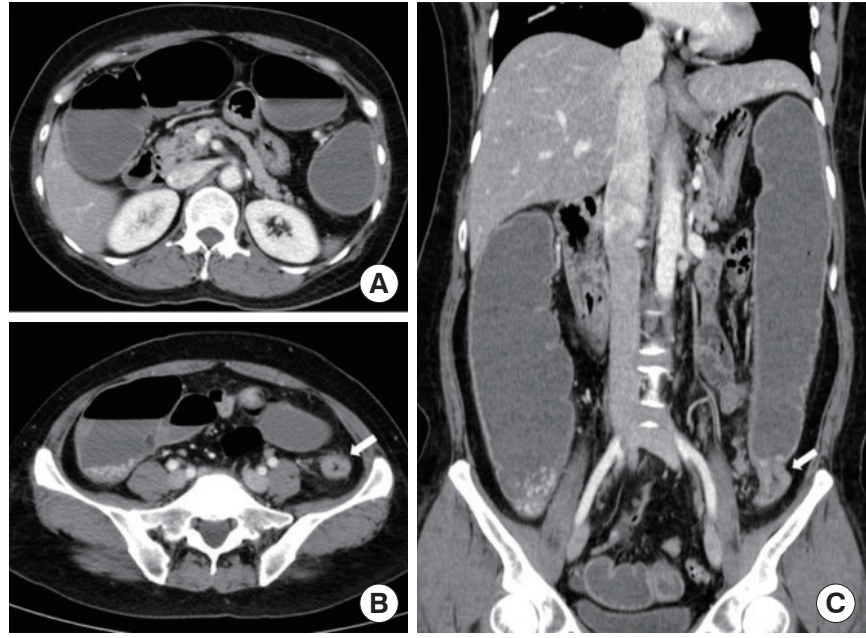

Fig. 1. Axial (A, B) and coronal (C) computed tomography of a 49-year-old female patient with obstructive descending colon cancer. A luminal obstructing mass of about 2 to $3 \mathrm{~cm}$ in length can be seen at the distal descending colon (arrow, B, C) and massively distended proximal colon (A-C).

performed in all cases with proximal colonic ischemia according to intraoperative findings. All patients were postoperatively offered a clear, oral, fluid diet after having passed bowel gas or having had a bowel movement and were progressively advanced to a soft diet and a regular diet as tolerated.

Endoluminal colonic wall stents were inserted using fluoroscopy alone, colonoscopy alone, or colonoscopy under fluoroscopy guidance. The method of colonic stenting was determined according to location of the obstructive cancer and the availability of an interventional radiologist. In the distal sigmoid colon, the SEMS (BONASTENT Colo-Rectal stents, Sewoon Medical Co., Cheonan, Korea, or Taewoong Niti-S Enteral colonic Stents, Taewoong Medical Co., Gimpo, Korea) was usually inserted using fluoroscopy alone or colonoscopy alone. For more proximal lesions, the SEMS was inserted using colonoscopy under fluoroscopy guidance. Following successful SEMS insertion, patients gradually received mechanical bowel preparation with low-dose polyethylene glycol after bowel movement had been confirmed. Preoperative colonoscopy through SEMS was attempted based on the surgeon's decision according to computed tomography findings. Colonoscopy within postoperative 6 months was recommended for patients who had undergone a colon segmental resection without preoperative complete colonoscopy. Surgery was usually scheduled within 7 days (median, 6 days; interquartile range, 5-6 days) of a technically successful SEMS insertion. In patients who experienced a technical failure of SEMS insertion, a subtotal colectomy was performed immediately by using an open or a laparoscopic technique.

Results are expressed as mean \pm standard deviation or median value with interquartile range. Continuous variables were compared using the Student t-test or the Mann-Whitney U-test. Dis- 
Table 1. Clinicopathological characteristics

\begin{tabular}{lccc}
\hline Variable & STC $(\mathrm{n}=24)$ & SEMS $(\mathrm{n}=70)$ & P-value \\
\hline Mean age (yr) & $71.8 \pm 12.4$ & $65.4 \pm 13.5$ & $0.045^{\mathrm{a}}$ \\
Sex. male : female & $12: 12$ & $46: 24$ & $0.172^{\mathrm{b}}$ \\
Underlying disease (\%) & $13(54.2)$ & $27(38.6)$ & $0.182^{\mathrm{b}}$ \\
ASA physical status & & & $0.344^{\mathrm{c}}$ \\
$\quad$ I or II & $18(75.0)$ & $60(85.7)$ & \\
$\quad$ III or IV & $6(25.0)$ & $10(14.3)$ & \\
Tumor location & & & $0.024^{\mathrm{b}}$ \\
$\quad$ Descending colon & $9(37.5)$ & $11(15.7)$ & \\
$\quad$ Sigmoid colon & $15(62.5)$ & $59(84.3)$ & \\
Pathologic stage & & & $0.691^{\mathrm{b}}$ \\
I & $0(0)$ & $0(0)$ & \\
II & $10(41.7)$ & $23(32.9)$ & \\
III & $12(50.0)$ & $42(60.0)$ & \\
IV & $2(8.3)$ & $5(7.1)$ & \\
Synchronous neoplasms & $5(20.8)$ & $12(20.7)^{\mathrm{d}}$ & $1.000^{\mathrm{c}}$ \\
Malignancy & $1(4.2)$ & $0(0)^{\mathrm{d}}$ & $0.292^{\mathrm{c}}$ \\
Advanced adenoma & $4(16.7)$ & $12(20.7)^{\mathrm{d}}$ & $0.768^{\mathrm{c}}$ \\
\hline
\end{tabular}

Values are presented as mean \pm standard deviation or number (\%).

STC, subtotal colectomy; SEMS, self-expandable metallic stent as a bridge to colectomy; ASA, American Society of Anesthesiologists.

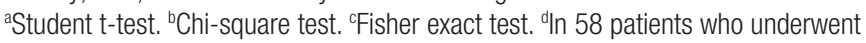
perioperative complete colonoscopy.

crete variables were analyzed with the chi-square test or the Fisher exact test. Statistical analyses were performed using a IBM SPSS Statistics ver. 19.0 (IBM Co., Armonk, NY, USA). P-values < 0.05 were considered statistically significant.

\section{RESULTS}

A subtotal colectomy was attempted in 24 patients and preoperative SEMS insertion was attempted in 70 patients. SEMS insertion technically failed in 5 patients (7.1\%): 3 complete obstructions that the guide wire could not pass through, one incorrect position, and one perforation. All 5 patients with SEMS technical failure underwent a subtotal colectomy. Outcomes were analyzed on an intention-to-treat basis. Clinicopathological characteristics are shown in Table 1. Mean age and rate of obstruction in the descending colon were higher in the subtotal colectomy group than the SEMS group. Sex, underlying disease, American Society of Anesthesiologists physical status, and pathological stage showed no significant differences among groups.

Differences in operative and perioperative outcomes are shown in Table 2. The median interval to surgery after SEMS insertion was 5 days. Laparoscopic surgery was more frequently performed in patients in the SEMS group (62 of 70, 88.6\%). A laparoscopic subtotal colectomy was performed in 4 of 24 patients (16.7\%).
Table 2. Operative and postoperative outcomes

\begin{tabular}{|c|c|c|c|}
\hline Variable & $\begin{array}{c}\text { STC } \\
(n=24)\end{array}$ & $\begin{array}{c}\text { SEMS } \\
(n=70)\end{array}$ & P-value \\
\hline Method of surgery & & & $<0.001^{\mathrm{a}}$ \\
\hline Laparoscopic & $4(16.7)$ & $62(88.6)$ & \\
\hline Open & $20(83.3)$ & $8(11.4)$ & \\
\hline Type of surgery & & & $<0.001^{\mathrm{a}}$ \\
\hline STC & 24 & 5 & \\
\hline Left hemicolectomy & 0 & 11 & \\
\hline Anterior resection & 0 & 59 & \\
\hline Length of surgery (hr) & $183.1 \pm 42.9$ & $146.1 \pm 45.1$ & $0.001^{b}$ \\
\hline Morbidity (No. of patients) & $2(8.3)$ & $22(31.4)$ & $0.025^{\mathrm{a}}$ \\
\hline lleus & $1(4.2)$ & $11(15.7)$ & $0.285^{c}$ \\
\hline Pulmonary complications & $1(4.2)$ & $8(11.4)$ & $0.439^{c}$ \\
\hline Urinary tract injury & $0(0)$ & $3(4.3)$ & $0.568^{c}$ \\
\hline Brachial plexus injury & $0(0)$ & $1(1.4)$ & $1.000^{\circ}$ \\
\hline Wound complications & $0(0)$ & $1(1.4)$ & $1.000^{c}$ \\
\hline Sepsis & $0(0)$ & $2(2.9)$ & $1.000^{c}$ \\
\hline Mortality & $0(0)$ & $2(2.9)$ & $1.000^{c}$ \\
\hline $\begin{array}{l}\text { Time to tolerance of regular diet } \\
\text { (day) }\end{array}$ & $6(5-7)$ & $4(3-6)$ & $<0.001^{d}$ \\
\hline Total hospital stay (day) & $12(10-15)$ & $16(13-19)$ & $<0.001^{d}$ \\
\hline Postoperative hospital stay (day) & $11(9-13)$ & $9(8-13)$ & $0.125^{d}$ \\
\hline $\begin{array}{l}\text { Bowel movements at postoperative } \\
3-6 \text { months }\end{array}$ & $2(2-3)$ & $1(1-2)$ & $<0.001^{d}$ \\
\hline $\begin{array}{l}\text { Antidiarrheal medication at post- } \\
\text { operative 3-6 months }\end{array}$ & $6(25.0)$ & $6(8.8)$ & $0.072^{c}$ \\
\hline
\end{tabular}

Values are presented as number (\%), mean \pm standard deviation, or median (interquartile range).

STC, subtotal colectomy; SEMS, self-expandable metallic stent as a bridge to colectomy.

${ }^{a}$ Chi-square test. ${ }^{b}$ Student t-test. ${ }^{\circ}$ Fisher exact test. ${ }^{d}$ Mann-Whitney U-test.

The type of laparoscopic surgery was usually hand-assisted laparoscopic surgery (HALS). Conventional laparoscopic surgery was performed in 3 cases: 1 subtotal colectomy and 2 SEMS cases. The length of surgery was shorter in the SEMS group than the subtotal colectomy group.

The overall rate of perioperative morbidity was higher in the SEMS group. No Clavien-Dindo grade III or IV complications were observed in the subtotal colectomy group, but 2 septic complications (2.9\%) occurred in the SEMS group. These 2 patients, who had undergone SEMS insertion that failed because of complete obstruction, died from sepsis. Intraoperative findings showed intestinal ischemia from a massively dilated proximal colon in these patients. These 2 patients underwent subtotal colectomy, but postoperative septic complications developed, possibly due to bacterial translocation and bacteremia from a massively 
dilated proximal colon. Other postoperative complications observed in the SEMS group were Clavien-Dindo grade I or II. Three cases of intraoperative ureteral injuries occurred in the SEMS group, but all ureteral injuries were recognized and repaired during the surgery. Prolonged postoperative ileus, which is defined as intolerance to a soft diet longer than 6 days after surgery, tended to be more frequent in the SEMS group than the subtotal colectomy group, although the difference was not significant.

The total hospital stay was longer in the SEMS group than the subtotal colectomy group, even though time to tolerance of regular diet was shorter. The number of bowel movements was increased in the subtotal colectomy group, and the incidence of antidiarrheal medication at postoperative 3-6 months was $25 \%$ (6 of 24). The median number of bowel movements in subtotal colectomy group was twice (interquartile range, 2-3) per day.

One patient $(4.2 \%)$ in the subtotal colectomy group had synchronous cancer at the hepatic flexure. Sigmoidoscopy was preoperatively performed in 16 of 24 patients (66.7\%) in the subtotal colectomy group; no synchronous cancer or advanced adenoma (defined as greater than $1 \mathrm{~cm}$ in diameter and/or with a villous component and/or with severe dysplasia) was observed below the obstructive cancer. Five of 8 patients without preoperative sigmoidoscopy underwent postoperative sigmoidoscopy with no synchronous tumor being found in the distal colon and rectum. Preoperative colonoscopy was attempted in 24 of 65 patients (36.9\%) with successful SEMS insertion, and 17 of 24 patients (70.8\%) successfully received a complete colonoscopy through SEMS insertion; no synchronous cancer was observed in these patients, but an advanced adenoma occurred in 5 of 17 patients (29.4\%). A complete postoperative colonoscopy was performed in 41 of 48 patients (85.4\%) who had undergone a colon segmental resection following successful SEMS insertion without preoperative complete colonoscopy; no synchronous cancer was observed in these patients, but an advanced adenoma occurred in 2 of 41 patients (4.9\%).

\section{DISCUSSION}

The morbidity $(40 \%-50 \%)$ and the mortality $(15 \%-20 \%)$ rates for patients who undergo emergency colorectal surgery for acute malignant obstruction have been reported to be significantly higher than for patients who undergo elective colorectal cancer surgery $[17,18]$. Therefore, staged surgery is performed for left coloncancer obstructions to reduce the risk of peritonitis from fecal contamination caused by an unprepared bowel. The main advantage of staged surgery using colostomy is that it minimizes surgical trauma in patients with a precarious general condition. However, many patients, up to about $45 \%$, cannot undergo colostomy reversal because of their underlying general condition [19]. Therefore, many surgeons need safe and effective surgical techniques to achieve primary colon-cancer resection and restore bowel continuity in patients with obstructive left colon cancer.

Surgical strategies for primary resection of obstructive left colon cancer and anastomosis that have been developed and reported include intraoperative colonic lavage or preoperative placement of SEMS $[4,6]$. Reports have suggested the safety and effectiveness of SEMS use; however, the most favorable results are obtained by per-protocol analysis $[4,6,14]$. A prospective, randomized trial analyzed on an intention-to-treat basis failed to demonstrate that preoperative SEMS use for patients with left colon-cancer obstruction decreased the need for stoma placement because SEMS insertion was technically successful in only $47 \%$ of the patients [10]. Therefore, a preoperative SEMS procedure does not clearly have more advantageous outcomes than emergency surgery [20, $21]$. However, a recent randomized trial with a $70 \%$ successful stenting rate found that SEMS insertion followed by interval elective surgery may be safer than emergency surgery, with a trend towards lower morbidity and mortality [7]. To achieve favorable outcomes from preoperative SEMS insertion, the technical and the clinical success rates of using a SEMS procedure as a bridge to surgery must be increased. Colonic stenting is considered relatively safe, with a mortality rate of about $1 \%$ [22]. However, the reported frequency of perforation after colonic stenting is about $4 \%[22,23]$, and inadequate decompression, stent migration, bleeding and pain have been reported [24]. Few studies have investigated predictors of failed stenting. Kim et al. [8] suggested that the use of multiple SEMS appeared to be a risk factor for surgical failure (insufficient colonic decompression), but no risk factor was detected before attempting SEMS insertion. Because the location and the degree of obstruction and the shape or the length of the malignant stricture might be preprocedural risk factors for stenting failure, studies focusing on these risk factors should be performed in the future.

Another issue for SEMS insertion as a bridge to surgery is that the interval to surgery after SEMS insertion varies among studies because of the lack of strict guidelines for the timing of the elective surgery. The median interval to elective surgery has been reported to be $5-10$ days after SEMS insertion $[6,7,14]$. Longer periods of more than 14 days after SEMS insertion may increase dense fibrotic adhesions around the lesion, which might make the surgery more difficult [7]. In our study, elective surgery after SEMS insertion was performed as early as possible and only if the distended proximal colon had been decompressed and gradual mechanical bowel preparation had been completed. The total hospital stay was longer in the SEMS group than in the subtotal colectomy group because of the time for bowel preparation, even though the time to tolerance of a regular diet was shorter.

Laparoscopic surgery could be completed in 62 of 70 patients $(88.6 \%)$ in the SEMS group. Adhesion around lesions and bulky tumors deteriorated by SEMS insertion could be overcome using the HALS technique. SEMS insertion may increase the feasibility of laparoscopic surgery for patients with obstructive colorectal cancer because successful SEMS insertion allows for mechanical 
bowel preparation and decompression of the distended bowel to provide a laparoscopic surgical space. Preoperative SEMS insertion is not obviously safer or more effective than other surgical strategies; however, successful SEMS insertion certainly makes possible the completion of minimally invasive surgeries $[4,7]$.

A subtotal colectomy and ileosigmoid anastomosis is a 1-stage surgery for treating patients with obstructive left colon cancer. Mortality rates are reasonably acceptable at less than $10 \%$ based on previous reports $[5,16,25]$. In our study, morbidity was 2 of 24 $(8.3 \%)$, mortality was 0 of $24(0 \%)$, and postoperative bowel function was preserved as measured by the number of bowel movements. Previous studies found that about $12 \%-19 \%$ of patients needed antidiarrheal medication after a subtotal or a total colectomy [15, 25]. In our study, 6 of 24 patients $(25 \%)$ who had undergone a subtotal colectomy needed antidiarrheal medication at postoperative 3-6 months, and the median number of bowel movements was 2 (interquartile range, 2-3) per day. The median number of bowel movements in a previous study including patients who had undergone a total colectomy was 6 (range, 3-11) within the first 3 postoperative months, although the increase in bowel movements was temporary and tended to disappear after the sixth postoperative month [14]. Patients with rectal or rectosigmoid junction cancer were excluded in our present study. If patients need ileorectal anastomosis, we recommend other surgical strategies for segmental resection and anastomosis such as SEMS insertion or intraoperative colonic irrigation. Rectal or rectosigmoid junctions are theoretically easier to stent because they do not require passing the redundant colon, an especially difficult sigmoid segment [24].

Another advantage of a subtotal colectomy for treating patients with obstructive left colon cancer is risk reduction for those patients with proximal synchronous malignant lesions. About 3\%$4 \%$ of colorectal cancer patients have synchronous colorectal cancer [26, 27]. In our study, 1 patient (4.2\%) in the subtotal colectomy group had synchronous cancer; other studies have reported synchronous cancer in $3 \%-11 \%$ of patients who undergo a total or a subtotal colectomy for obstructive left colon cancer $[14,16]$. Preoperative colonoscopy with a conventional colonoscope through the colonic stent is possible in only $62.5 \%$ of patients [28]. Our study showed that complete colonoscopy through the SEMS was successful in $70.8 \%$ of patients. We observed no synchronous cancer in patients who had undergone a colon segmental resection following successful SEMS insertion, but a synchronous advanced adenoma was present preoperatively in $29.4 \%$ and postoperatively in $4.9 \%$. A subtotal colectomy might be an oncologically safe strategy in patients with a synchronous tumor.

Some reports have suggested that preoperative insertion of a SEMS might be associated with adverse oncologic outcomes. In one study, SEMS insertion had a significant adverse effect on 5 -year overall survival and 5-year disease-free survival compared with a nonobstructing elective surgery group [12]. A recent comparative study on patients with obstructive left colon cancer also showed that overall survival and 5-year overall survival were significantly lower and 5-year cancer-specific mortality was significantly higher in a SEMS group compared to a surgery-only group [13]. A study using CK20 mRNA levels in peripheral blood supported the dissemination of cancer cells into the peripheral circulation after endoscopic stent insertion [11]. Moreover, stenting has a high rate of failure, which includes perforation, and cancer perforation certainly jeopardizes oncological outcomes.

In conclusion, a subtotal colectomy for treating patients with obstructive left colon cancer is a clinically safer, 1-stage, surgical strategy compared to SEMS insertion as a bridge to surgery. A subtotal colectomy can treat synchronous tumors in the proximal colon, reduce total hospital stay, and avoid oncologic deterioration from colonic stenting, as bowel function is preserved. Preoperative SEMS insertion should be considered only for patients with rectal or rectosigmoid junction cancer and patients for whom minimally invasive surgery is planned.

\section{CONFLICT OF INTEREST}

No potential conflict of interest relevant to this article was reported.

\section{REFERENCES}

1. Deans GT, Krukowski ZH, Irwin ST. Malignant obstruction of the left colon. Br J Surg 1994;81:1270-6.

2. Biondo S, Parés D, Frago R, Martí-Ragué J, Kreisler E, De Oca J, et al. Large bowel obstruction: predictive factors for postoperative mortality. Dis Colon Rectum 2004;47:1889-97.

3. Käser SA, Glauser PM, Künzli B, Dolanc R, Bassotti G, Maurer CA. Subtotal colectomy for malignant left-sided colon obstruction is associated with a lower anastomotic leak rate than segmental colectomy. Anticancer Res 2012;32:3501-5.

4. Park IJ, Choi GS, Kang BM, Lim KH, Lee IT, Jeon SW, et al. Comparison of one-stage managements of obstructing left-sided colon and rectal cancer: stent-laparoscopic approach vs. intraoperative colonic lavage. J Gastrointest Surg 2009;13:960-5.

5. Torralba JA, Robles R, Parrilla P, Lujan JA, Liron R, Piñero A, et al. Subtotal colectomy vs. intraoperative colonic irrigation in the management of obstructed left colon carcinoma. Dis Colon Rectum 1998;41:18-22.

6. Alcántara M, Serra-Aracil X, Falcó J, Mora L, Bombardó J, Navarro S. Prospective, controlled, randomized study of intraoperative colonic lavage versus stent placement in obstructive left-sided colonic cancer. World J Surg 2011;35:1904-10.

7. Ho KS, Quah HM, Lim JF, Tang CL, Eu KW. Endoscopic stenting and elective surgery versus emergency surgery for left-sided malignant colonic obstruction: a prospective randomized trial. Int J Colorectal Dis 2012;27:355-62.

8. Kim JH, Kwon KA, Lee JJ, Lee WS, Baek JH, Kim YJ, et al. Surgical failure after colonic stenting as a bridge to surgery. World J 
Gastroenterol 2014;20:11826-34.

9. van Hooft JE, Bemelman WA, Oldenburg B, Marinelli AW, Lutke Holzik MF, Grubben MJ, et al. Colonic stenting versus emergency surgery for acute left-sided malignant colonic obstruction: a multicentre randomised trial. Lancet Oncol 2011;12:344-52.

10. Pirlet IA, Slim K, Kwiatkowski F, Michot F, Millat BL. Emergency preoperative stenting versus surgery for acute left-sided malignant colonic obstruction: a multicenter randomized controlled trial. Surg Endosc 2011;25:1814-21.

11. Maruthachalam K, Lash GE, Shenton BK, Horgan AF. Tumour cell dissemination following endoscopic stent insertion. Br J Surg 2007;94:1151-4.

12. Kim JS, Hur H, Min BS, Sohn SK, Cho CH, Kim NK. Oncologic outcomes of self-expanding metallic stent insertion as a bridge to surgery in the management of left-sided colon cancer obstruction: comparison with nonobstructing elective surgery. World J Surg 2009;33:1281-6.

13. Sabbagh C, Browet F, Diouf M, Cosse C, Brehant O, Bartoli E, et al. Is stenting as "a bridge to surgery" an oncologically safe strategy for the management of acute, left-sided, malignant, colonic obstruction? A comparative study with a propensity score analysis. Ann Surg 2013;258:107-15.

14. Ghazal AH, El-Shazly WG, Bessa SS, El-Riwini MT, Hussein AM. Colonic endolumenal stenting devices and elective surgery versus emergency subtotal/total colectomy in the management of malignant obstructed left colon carcinoma. J Gastrointest Surg 2013;17: 1123-9.

15. Manceau G, d'Annunzio E, Karoui M, Breton S, Rousseau G, Blanchet AS, et al. Elective subtotal colectomy with ileosigmoid anastomosis for colon cancer preserves bowel function and quality of life. Colorectal Dis 2013;15:1078-85.

16. Hennekinne-Mucci S, Tuech JJ, Bréhant O, Lermite E, Bergamaschi R, Pessaux P, et al. Emergency subtotal/total colectomy in the management of obstructed left colon carcinoma. Int J Colorectal Dis 2006;21:538-41.

17. Tekkis PP, Kinsman R, Thompson MR, Stamatakis JD; Association of Coloproctology of Great Britain, Ireland. The Association of Coloproctology of Great Britain and Ireland study of large bowel obstruction caused by colorectal cancer. Ann Surg 2004;
240:76-81.

18. Breitenstein S, Rickenbacher A, Berdajs D, Puhan M, Clavien PA, Demartines N. Systematic evaluation of surgical strategies for acute malignant left-sided colonic obstruction. Br J Surg 2007;94: 1451-60.

19. Pearce NW, Scott SD, Karran SJ. Timing and method of reversal of Hartmann's procedure. Br J Surg 1992;79:839-41.

20. Tan CJ, Dasari BV, Gardiner K. Systematic review and meta-analysis of randomized clinical trials of self-expanding metallic stents as a bridge to surgery versus emergency surgery for malignant left-sided large bowel obstruction. Br J Surg 2012;99:469-76.

21. Ye GY, Cui Z, Chen L, Zhong M. Colonic stenting vs emergent surgery for acute left-sided malignant colonic obstruction: a systematic review and meta-analysis. World J Gastroenterol 2012;18: 5608-15.

22. Khot UP, Lang AW, Murali K, Parker MC. Systematic review of the efficacy and safety of colorectal stents. Br J Surg 2002;89:1096102.

23. Sebastian S, Johnston S, Geoghegan T, Torreggiani W, Buckley M. Pooled analysis of the efficacy and safety of self-expanding metal stenting in malignant colorectal obstruction. Am J Gastroenterol 2004;99:2051-7.

24. Han SH, Lee JH. Colonic stent-related complications and their management. Clin Endosc 2014;47:415-9.

25. Stephenson BM, Shandall AA, Farouk R, Griffith G. Malignant left-sided large bowel obstruction managed by subtotal/total colectomy. Br J Surg 1990;77:1098-102.

26. Mulder SA, Kranse R, Damhuis RA, de Wilt JH, Ouwendijk RJ, Kuipers EJ, et al. Prevalence and prognosis of synchronous colorectal cancer: a Dutch population-based study. Cancer Epidemiol 2011;35:442-7.

27. Passman MA, Pommier RF, Vetto JT. Synchronous colon primaries have the same prognosis as solitary colon cancers. Dis Colon Rectum 1996;39:329-34.

28. Kim JS, Lee KM, Kim SW, Kim EJ, Lim CH, Oh ST, et al. Preoperative colonoscopy through the colonic stent in patients with colorectal cancer obstruction. World J Gastroenterol 2014;20: 10570-6. 